\title{
Preclinical Evaluation of Robotic-Assisted Sentinel Lymph Node Fluorescence Imaging
}

\author{
Michael A. Liss ${ }^{1,2}$, Salman Farshchi-Heydari ${ }^{3,4}$, Zhengtao Qin $^{3-5}$, Sean A. Hickey ${ }^{6}$, David J. Hall ${ }^{3,4}$, \\ Christopher J. Kane ${ }^{1,2,6}$, and David R. Vera ${ }^{2-4,6}$ \\ ${ }^{I}$ Department of Urology, University of California, San Diego, La Jolla, California; ${ }^{2}$ UCSD Moores Cancer Center. University of \\ California, San Diego, La Jolla, California; ${ }^{3}$ Department of Radiology, University of California, San Diego, La Jolla, California; \\ ${ }^{4}$ UCSD In Vivo Cancer and Molecular Imaging Center, University of California, San Diego, La Jolla, California; ${ }^{5}$ Department of \\ Chemistry and Biochemistry, University of California, San Diego, La Jolla, California; and ${ }^{6}$ Department of Surgery, University of \\ California, San Diego, La Jolla, California
}

\begin{abstract}
An ideal substance to provide convenient and accurate targeting for sentinel lymph node (SLN) mapping during robotic-assisted surgery has yet to be found. We used an animal model to determine the ability of the FireFly camera system to detect fluorescent SLNs after administration of a dual-labeled molecular imaging agent. Methods: We injected the footpads of New Zealand White rabbits with 1.7 or $8.4 \mathrm{nmol}$ of tilmanocept labeled with ${ }^{99 \mathrm{~m} T c}$ and a near-infrared fluorophore, IRDye $800 \mathrm{CW}$. One and $36 \mathrm{~h}$ after injection, popliteal lymph nodes, representing the SLNs, were dissected with the assistance of the FireFly camera system, a fluorescence-capable endoscopic imaging system. After excision of the paraaortic lymph nodes, which represented non-SLNs, we assayed all lymph nodes for radioactivity and fluorescence intensity. Results: Fluorescence within all popliteal lymph nodes was easily detected by the FireFly camera system. Fluorescence within the lymph channel could be imaged during the 1 -h studies. When compared with the paraaortic lymph nodes, the popliteal lymph nodes retain greater than $95 \%$ of the radioactivity at both 1 and $36 \mathrm{~h}$ after injection. At both doses (1.7 and $8.4 \mathrm{nmol})$, the popliteal nodes had higher $(P<0.050)$ optical fluorescence intensity than the paraaortic nodes at the 1- and 36-h time points. Conclusion: The FireFly camera system can easily detect tilmanocept labeled with a near-infrared fluorophore at least $36 \mathrm{~h}$ after administration. This ability will permit image acquisition and subsequent verification of fluorescence-labeled SLNs during robotic-assisted surgery.
\end{abstract}

Key Words: fluorescent tilmanocept; sentinel lymph node mapping; robotic-assisted surgery

J Nucl Med 2014; 55:1552-1556

DOI: 10.2967/jnumed.114.140871

$\mathbf{S}$

entinel lymph node (SLN) detection and excision is becoming incorporated in breast cancer and melanoma surgery $(1,2)$. However, there has been less progress with genitourinary cancers largely because of the limitations in available agents with favorable timing parameters from injection to imaging and surgery. For example, prostate cancer has considerable variation regarding the decision

Received Mar. 30, 2014; revision accepted May 5, 2014.

For correspondence or reprints contact: David R. Vera, UCSD Moores Cancer Center, 3855 Health Sciences Dr., La Jolla, CA 92093.

E-mail: dvera@ucsd.edu

Published online Jul. 14, 2014.

COPYRIGHT (c) 2014 by the Society of Nuclear Medicine and Molecular Imaging, Inc. to perform, or the extent of, pelvic lymph node dissection based on preoperative risk factors (3-5). The previously limited pelvic lymph node dissection usually does not include high-risk nodes, causing the current risk of positive lymph nodes to be lower than predicted (5). SLN biopsy in prostate cancer has been shown to accurately predict positive lymph nodes and, pending further research, may parallel the excellent results of SLN biopsy in breast cancer and melanoma $(6,7)$.

Limitations in previous studies include logistics and cumbersome intraoperative $\gamma$ detectors. However, the endoscope used in minimally invasive surgery has provided a platform for fluorescenceguided surgery. The United States Food and Drug Administration approved ${ }^{99 \mathrm{~m} T c-l a b e l e d}$ tilmanocept $(8)$, which is the first receptor-targeted radiopharmaceutical approved for lymphatic mapping (9-12). The benefits of receptor targeting allow the agent to bind and remain within SLN, providing logistic flexibility. Radiolabeled tilmanocept can be used to provide a preoperative roadmap using imaging with SPECT/CT (11) or PET/CT (13); however, a laparoscopic $\gamma$ detector would still be necessary (14). The ideal agent for robotic surgery would be the use of tilmanocept also labeled with a fluorophore for intraoperative visualization by the currently used FireFly endoscope (Da Vinci Si surgical robot; Intuitive Surgical) and the option of preoperative imaging.

In preparation for a phase 1 clinical trial, we investigated the use of fluorescence-labeled tilmanocept for SLN imaging using the FireFly endoscope. Our primary goal was to confirm the ability of the robotic camera to visualize the fluorescence accumulated within the SLN. We tested this ability at different molar doses of fluorescent tilmanocept and at different times after administration. Confirmation of SLN fluorescence imaging by the FireFly camera will provide us with the approximate molar dose and timing parameters with which to perform robotic-assisted fluorescence-based mapping of pelvic SLNs many hours after an ultrasound-guided transrectal injection of fluorescence-labeled tilmanocept into the canine prostate gland.

\section{MATERIALS AND METHODS}

\section{Experimental Design}

These experiments were designed to test the ability of the Da Vinci Si FireFly camera system to detect fluorescent SLNs resulting from the accumulation of $800 \mathrm{CW}$-tilmanocept. Our goal was to determine the amount of fluorescence-labeled tilmanocept that could be detected by the FireFly system but would not saturate the SLN and carry fluorescence to downstream lymph nodes, which would be falsely identified 
as SLNs. We elected to use a rabbit model that was used during the development of ${ }^{99 \mathrm{~m}} \mathrm{Tc}$-tilmanocept (15-17). In this model, a hind-limb administration of the fluorescence-labeled radiotracer would define the popliteal lymph node as the sentinel node and the paraaortic lymph nodes as the downstream lymph nodes. At a predetermined time point, the rabbits were euthanized before fluorescence-guided dissection using the FireFly endoscope attached to the Da Vinci Si robotic surgical system. Images were acquired with the standard clinical software and a modification that permitted the visualization of only the green fluorescence image in the operator console of the surgical robot. This modification, which will be incorporated into future versions of the da Vinci software, essentially turned off the white light of the camera system. After euthanasia, images were acquired over the popliteal fossa before surgical incision and after surgical exposure, when the SLN can be viewed without attenuation by overlying tissue. Four rabbits were used for each of 2 dose levels ( 1.7 and $8.4 \mathrm{nmol}$ ) and each of 2 imaging time points ( 1 and $36 \mathrm{~h}$ ), for a total of 16 rabbits. After euthanasia, the popliteal and paraaortic lymph nodes were excised via open dissection.

\section{Imaging Agent Preparation and Administration}

The preparation and quality control of ${ }^{99} \mathrm{~m}$ Tc-labeled $800 \mathrm{CW}$-tilmanocept was performed using a previously described method (18). The $800 \mathrm{CW}-$ tilmanocept used in this study consisted of an average of 1.5 IRDye $800 \mathrm{CW}$ molecules covalently attached to each tilmanocept molecule. The resulting molecular weight was approximately $18,000 \mathrm{~g} / \mathrm{mol}$. Quality control before each animal study was performed using high-performance liquid chromatography and instant thin-layer chromatography; both methods used fluorescence and radioactivity detection. Acceptance criteria required that both the radiochemical purity and the fluorescence purity be greater than $97 \%$.

Our animal protocol was approved by the University of California, San Diego, Institutional Animal Care and Use Committee, and the research staff was appropriately trained. We anesthetized New Zealand White rabbits with ketamine (Fort Dodge Animal Health) and xylazine (Vedco Inc.). The 0.10-mL injections each contained 3.7-7.4 MBq

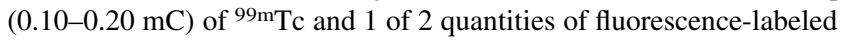
tilmanocept (1.7 or $8.4 \mathrm{nmol}$ ). Each volume was administered to both hind footpads of each rabbit with a 28 -gauge needle. We monitored the rabbits after anesthesia visually and with an oximeter that measures heart rate and functional oxygen saturation.

\section{FireFly Fluorescence Imaging Endoscope}

The FireFly fluorescence imaging endoscope is an optional component of the DaVinci Si robotic-assisted surgical system. The endoscope uses near-infrared light with an 805 -nm-wavelength excitation laser source. We investigated the ideal visualization characteristics of $800 \mathrm{CW}$ tilmanocept by turning off the background white light of the FireFly system. We also tested the detection ability of the camera system using the standard clinical firmware and hardware.

\section{Nuclear Counting}

Each node was placed in a plastic scintillation vial and assayed for radioactivity using a $\gamma$ well counter (100- to $200-\mathrm{keV}$ energy window, Gamma 9000; Beckman Instruments). The nodes were compared with a counting standard that was prepared from a known dilution of the injected material. The accumulation of ${ }^{99 \mathrm{~m}} \mathrm{Tc}-800 \mathrm{CW}$-tilmanocept within each SLN was expressed as percentage injected dose, which was calculated by comparing the tissue counts with the counting standard. The amount of fluorescent dye, IRDye $800 \mathrm{CW}$, was calculated by multiplying the percentage injected dose times the number of dyes per tilmanocept times the amount of $800 \mathrm{CW}$-tilmanocept administered to each hind paw times 0.01 . The percentage extraction $E_{S L N}$ of popliteal lymph nodes for each animal was calculated by Equation 1, where the $c p m_{L P}, c p m_{R P}$, and $c_{p m}$ represent the counts per minute within the left popliteal, right popliteal, and paraaortic lymph nodes, respectively.

$$
E_{S L N}=\frac{100\left(c p m_{L P}+c p m_{R P}-c p m_{P A}\right)}{c p m_{L P}+c p m_{R P}+c p m_{P A}}
$$

\section{Fluorescence Imaging}

Ex vivo optical imaging was performed with a time-correlated singlephoton fluorescence imaging system (Optix MX2; Advanced Research Technologies). Images were acquired for each excised lymph node using a scan resolution of $1 \mathrm{~mm}^{2}$ and a 758-nm pulsed laser diode with an $800-\mathrm{MHz}$ frequency and a $0.3-\mathrm{s}$ integration time. Emitted photons were collected through a pair of 780-nm long-pass filters and a 782-nm band-pass filter, both with a $20-\mathrm{nm}$ bandwidth. For each node, the total fluorescence intensity (counts per minute) was determined by an operator-defined region of interest and reported in kilocounts per second per microwatt $\left(\mathrm{kcts} \cdot \mathrm{s}^{-1} \cdot \mu \mathrm{W}^{-1}\right)$.

\section{Statistical Analysis}

The Friedman test for nonparametric paired samples (right popliteal, left popliteal, and paraaortic lymph nodes) was used, with a $P$ value of less than 0.05 needed for statistical significance. Data were analyzed using the SPSS statistical package (version 21; IBM) and reported as mean $\pm \mathrm{SD}$.

\section{RESULTS}

\section{Visualization of Fluorescent SLNs}

The FireFly endoscope attached to the Da Vinci Si robotic surgical system visualized all of the popliteal lymph nodes at 1 and $36 \mathrm{~h}$ after administration of 1.7 or $8.4 \mathrm{nmol}$ of fluorescence-labeled tilmanocept. Fluorescence visualization of all lymph nodes was possible with the FireFly endoscope using both the factory settings and the modified software, which turned off the white-light image at the viewing consol. Visualization of afferent lymph channels was possible $1 \mathrm{~h}$ after injection. Surgical exposure was not required for visualization of the SLN fluorescence when the duration between imaging and injection was $36 \mathrm{~h}$. Four lymph nodes of the 1-h studies were also visualized behind the muscle layer without the need for surgical exposure.

Figure 1 demonstrates the fluorescence detection of an SLN and its afferent lymph channels by the FireFly camera. This was accomplished at the lower of the 2 doses, $1.7 \mathrm{nmol}$ of fluorescencelabeled tilmanocept, and without surgical exposure of the lymph node or the afferent lymph channels. Figure $1 \mathrm{~A}$ is a bright-field image of a rabbit hind-limb acquired $1 \mathrm{~h}$ after a 1.7-nmol injection of 800CWtilmanocept. After the switch to fluorescence mode (Fig. 1B), fluorescence could be visualized traveling up the lymphatic channel (arrow) to the SLN located in the popliteal fossa (arrow in Fig. $1 C$ ). Figure 1D is a fluorescence-mode image of the SLN (arrow), which accumulated 15 pmol of fluorescence-labeled tilmanocept to which 25 pmol of IRDye $800 \mathrm{CW}$ was covalently attached. After excision, the SLN produced a fluorescence intensity of 8.6 $\mathrm{kcts} \cdot \mathrm{s}^{-1} \cdot \mu \mathrm{W}^{-1}$. The paraaortic lymph nodes contained $0.14 \mathrm{pmol}$ of $800 \mathrm{CW}$-tilmanocept $(0.20 \mathrm{pmol}$ IRDye $800 \mathrm{CW})$, which produced a fluorescence intensity of $0.16 \mathrm{kcts} \cdot \mathrm{s}^{-1} \cdot \mu \mathrm{W}^{-1}$. The percentage extraction was $98.3 \%$. The fluorescence images (Figs. 1B and 1D) use a modification of the console software that allows visualization of only the fluorescence image from the FireFly camera.

\section{SLN Accumulation}

Table 1 lists the means and relative SDs of the various measurements of SLN accumulation - percentage injected dose, amount of fluorescent dye, percentage extraction, and fluorescence intensity-for each of the experimental groups $(1 \mathrm{~h}$ after a $1.7-\mathrm{nmol} 800 \mathrm{CW}$-tilmanocept 

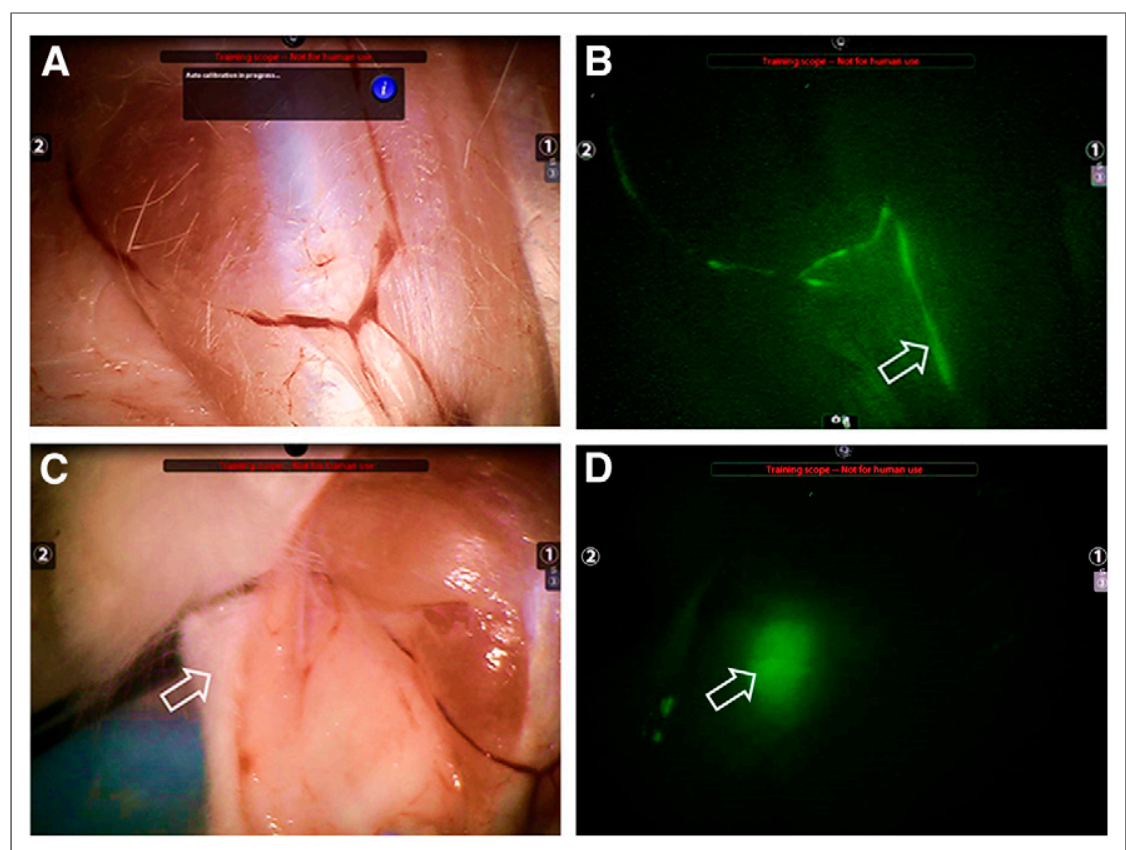

FIGURE 1. The FireFly endoscope attached to Da Vinci Si robotic surgical system visualized all popliteal lymph nodes at 1 and $36 \mathrm{~h}$ after administration of 1.7 or $8.4 \mathrm{nmol}$ of $99 \mathrm{~m} T \mathrm{Tc}$-labeled fluorescent tilmanocept. (A) Bright-field image of rabbit hind-limb was acquired $1 \mathrm{~h}$ after $1.7-\mathrm{nmol}$ injection of $800 \mathrm{CW}$-tilmanocept. (B) After switch to fluorescence mode, lymphatic channel (arrow) was visualized. (C and $D$ ) On repositioning of camera to visualize popliteal region (arrow, $C$ ), fluorescence mode visualized SLN (arrow, D) without surgical exposure. SLN accumulated $0.87 \%$ of injected dose $(25 \mathrm{pmol}$ of IRDye $800 \mathrm{CW})$.

injection, $36 \mathrm{~h}$ after a 1.7-mol injection, $1 \mathrm{~h}$ after an 8.4-nmol injection, and $36 \mathrm{~h}$ after an 8.4-nmol injection).

SLN accumulation was higher at $1 \mathrm{~h}$ after injection than at the later time point. At $1 \mathrm{~h}$, the mean and SD of the percentage injected dose from the 8 SLNs receiving the $1.7-\mathrm{nmol}$ dose of $800 \mathrm{CW}$ tilmanocept was $0.95 \% \pm 0.78 \%$; at $36 \mathrm{~h}$ the percentage injected dose was $0.56 \% \pm 0.39 \%$. The $8.4-\mathrm{nmol}$ dose delivered $1.64 \% \pm$ $0.77 \%$ at $1 \mathrm{~h}$ and $1.21 \% \pm 0.33 \%$ at $36 \mathrm{~h}$. 800CW-tilmanocept delivered more fluorescent dye using the higher dose $(8.4 \mathrm{nmol})$ at $1 \mathrm{~h}$ from injection $(0.21 \pm 0.10$ vs. $0.024 \pm 0.020 \mathrm{pmol} ; P<0.05)$ and at $36 \mathrm{~h}$ after injection $(0.15 \pm 0.04$ vs. $0.014 \pm 0.0 .010 \mathrm{pmol}$; $P<0.05)$. The fluorescence intensity of all the paraaortic nodes was less than $10 \%$ of the popliteal lymph nodes. At both doses (1.7 and $8.4 \mathrm{nmol})$, the popliteal nodes had higher $(P<0.050)$ optical fluorescence intensity than the paraaortic nodes at the $1-$ and $36-\mathrm{h}$ time points. The percentage SLN extraction for all the experimental groups was greater than $95 \%$, indicating the lack of SLN saturation at both low and high doses (1.7 and $8.4 \mathrm{nmol})$ of $800 \mathrm{CW}$-tilmanocept. The 8.4-nmol dose produced higher fluorescence intensity with the SLNs at both time points: $19.1 \pm 9.23 \mathrm{kcts} \cdot \mathrm{s}^{-1} \cdot \mu \mathrm{W}^{-1} 1 \mathrm{~h}$ after injection and $11.6 \pm 4.24 \mathrm{kcts} \cdot \mathrm{s}^{-1} \cdot \mu \mathrm{W}^{-1} 36 \mathrm{~h}$ after injection. The 1.7-nmol dose of $800 \mathrm{CW}$-tilmanocept produced a fluorescence intensity of $6.22 \pm 2.45 \mathrm{kcts} \cdot \mathrm{s}^{-1} \cdot \mu \mathrm{W}^{-1}$ at $1 \mathrm{~h}$ and $8.62 \pm 3.72$ $\mathrm{kcts} \cdot \mathrm{s}^{-1} \cdot \mu \mathrm{W}^{-1}$ at $36 \mathrm{~h}$. Increasing the amount of $800 \mathrm{CW}$-tilmanocept injected produced a significantly higher $(P<0.05)$ fluorescence intensity at both time points.

\section{DISCUSSION}

This study demonstrated that the FireFly endoscopic camera system of the Da Vinci Si surgical system can image fluorescent SLNs resulting from an 8.4-nmol injection of $800 \mathrm{CW}$-tilmanocept and that this can be accomplished without receptor saturation within the SLN. Moreover, when imaging is performed at the appropriate time, the FireFly camera can visualize fluorescence from afferent lymph channels.

Previous animal studies using the New Zealand White rabbit model have shown that without a molecular or physiologic mechanism to retain the imaging agent within the lymph node, nearly all ${ }^{99 \mathrm{~m}} \mathrm{Tc}$ had passed through the SLNs by $6 \mathrm{~h}$ after injection (15-17). Using 99m Tc-labeled $800 \mathrm{CW}$-tilmanocept, we show that radioactivity levels can be detected in popliteal lymph nodes at 1 and $36 \mathrm{~h}$ after footpad injection in a rabbit model. The molecule is retained within the popliteal lymph node and does not significantly migrate to the next level of lymph nodes (paraaortic) or significantly dissipate over time. These results suggest that the receptor binds within $1 \mathrm{~h}$ and will stay localized up to $36 \mathrm{~h}$, allowing for flexibility in image acquisition with nuclear medicine scanning before surgery. The use of imaging before surgery to identify SLNs has been shown to reduce operative time when compared with $\gamma$ probe-guided lymph node dissections during radical prostatectomy $(19,20)$.

Pelvic lymphadenectomy during radical prostatectomy provides staging information that may guide adjuvant therapy (21-25). The extent and definition of the pelvic lymph node dissection are controversial $(5,26,27)$. Studies have shown that limited pelvic lymph node dissection resects only $35 \%$ of the primary landing zones of prostate-draining lymph nodes (28). Therefore, the SLN dissection has become an emerging concept during radical prostatectomy to tailor surgery and decrease the risk-to-benefit ratio of lymph node dissection by resecting only high-yield nodes $(7,29,30)$. Moreover, this technique may reduce the complications of increasingly extensive lymph node dissections (i.e., lymphocele, lymphedema, deep venous thrombosis, and pulmonary embolism) (31). One of the initial studies of SLN dissection was performed by Wawroschek et al. by injecting ${ }^{99 \mathrm{~m}} \mathrm{Tc}$-labeled colloid into the prostate and performing dynamic lymphoscintigraphy followed by surgery with $\gamma$-probe guidance the next day (29). This concept was later confirmed in a study with over 1,000 prostatectomies. That study noted that only $1 \%$ of metastases were found in patients with negative SLNs; however, the study did not provide intraoperative visual confirmation with fluorescence (32).

Previous investigations have added indocyanine green (ICG) to fluorescently image lymph channels and SLNs. One study used ${ }^{99 \mathrm{~m}}$ Tc-labeled Nanocoll (GE Healthcare) to image before radical prostatectomy followed by a separate ICG injection at the time of surgery (33). A separate study used a mixture of ICG and ${ }^{99 \mathrm{~m}} \mathrm{Tc}-$ labeled Nanocoll imaged by SPECT/CT $2 \mathrm{~h}$ after injection, followed by surgery within the hour (20). The use of an ICG-radiolabeled Nanocoll mixture assumes that the dye binds to Nanocoll with adequate affinity for valid SLN mapping. Both studies using ICG detection showed accurate results (34); however, the use of ICG 
TABLE 1

SLN Accumulation

\begin{tabular}{|c|c|c|c|c|c|c|c|}
\hline $\begin{array}{l}\text { Number } \\
\text { of SLNs }\end{array}$ & $\begin{array}{l}\text { Location } \\
\text { of SLNs }\end{array}$ & $\begin{array}{l}\text { Time } \\
\text { (h) }\end{array}$ & $\begin{array}{l}\text { Tilmanocept } \\
\text { injected (nmol) }\end{array}$ & $\begin{array}{l}\text { Percentage } \\
\text { injected dose }\end{array}$ & $\begin{array}{l}\text { IRDye800CW } \\
\text { (nmol) }\end{array}$ & $\begin{array}{l}\text { Extraction } \\
(\%)^{*}\end{array}$ & $\begin{array}{l}\text { Fluorescence intensity } \\
\qquad\left(\text { kcts s }^{-1} \mu \mathrm{W}^{-1}\right)^{\dagger}\end{array}$ \\
\hline 8 & Popliteal & 1.0 & 1.7 & $0.95 \pm 0.78$ & $0.024 \pm 0.020^{\ddagger}$ & $99.4 \pm 4.0$ & $6.22 \pm 2.45^{\ddagger}$ \\
\hline 8 & Popliteal & 36.0 & 1.7 & $0.56 \pm 0.39 \ddagger$ & $0.014 \pm 0.010^{\ddagger}$ & $98.5 \pm 0.9$ & $8.62 \pm 3.72^{\ddagger}$ \\
\hline 8 & Popliteal & 1.0 & 8.4 & $1.64 \pm 0.77$ & $0.21 \pm 0.10$ & $95.1 \pm 5.2$ & $19.1 \pm 9.23$ \\
\hline 8 & Popliteal & 36.0 & 8.4 & $1.21 \pm 0.33$ & $0.15 \pm 0.04$ & $94.2 \pm 5.9$ & $11.6 \pm 4.24$ \\
\hline \multicolumn{8}{|c|}{$\begin{array}{l}{ }^{*} \text { Measured by y well counter }(100-200 \mathrm{keV}) \text {. } \\
{ }^{\dagger} \text { Measured by Optix MX2. } \\
{ }^{\ddagger} P<0.05 \text { for comparison with data from same time but different amount of injected tilmanocept. } \\
\text { Data are mean } \pm S D \text {. }\end{array}$} \\
\hline
\end{tabular}

in this manner requires multiple injections or an extra compounding step by a pharmacist. Moreover, Nanocoll is prepared from human blood and can have similar risks to transfusion. Lastly, Nanocoll is not available in the United States. Therefore, using a non-blood-based dual-labeled receptor-targeted molecule such as $800 \mathrm{CW}$-tilmanocept may have better logistic properties with reduced risk. In addition to determining the benefit of the 36-h interval, we also established that the larger dose $(8.4 \mathrm{nmol})$ provided improved fluorescence without saturation and migration to next-level lymph nodes.

\section{CONCLUSION}

${ }^{99 m}$ Tc-tilmanocept covalently tagged with IRDye $800 \mathrm{CW}$ can provide fluorescence detection of SLNs by the FireFly endoscope used during robotic surgery. Detection can be achieved with and without background white light. The molecule remains in the SLN at the 2 tested doses for at least $36 \mathrm{~h}$ to allow image acquisition and subsequent surgical verification via fluorescence guidance. Further in vivo (35) and subsequent human studies are needed to provide more information on the feasibility and accuracy of this technology during robotic surgery.

\section{DISCLOSURE}

The costs of publication of this article were defrayed in part by the payment of page charges. Therefore, and solely to indicate this fact, this article is hereby marked "advertisement" in accordance with 18 USC section 1734. This research was supported by the National Cancer Institute (P50 CA114745, P30 CA023100) and Intuitive Surgical. Intuitive provided software modifications to the Da Vinci Si robotic system and the FireFly endoscope adjustments. Otherwise they were not involved in the design, analysis, or writing of this article. Dr. Vera is the inventor of tilmanocept. Dr. Kane is a consultant or has received honoraria from Intuitive Surgical Inc. No other potential conflict of interest relevant to this article was reported.

\section{ACKNOWLEDGMENTS}

We thank LiCOR Biosciences for supplying the IRDye 800CW NHS-ester dye and Dr. Jonathan Sorger of Intuitive Surgical for his assistance with software modifications of the Da Vinci Si robotic surgical system.

\section{REFERENCES}

1. Krag DN, Anderson SJ, Julian TB, et al. Sentinel-lymph-node resection compared with conventional axillary-lymph-node dissection in clinically node-negative patients with breast cancer: overall survival findings from the NSABP B-32 randomised phase 3 trial. Lancet Oncol. 2010;11:927-933.

2. Valsecchi ME, Silbermins D, de Rosa N, Wong SL, Lyman GH. Lymphatic mapping and sentinel lymph node biopsy in patients with melanoma: a metaanalysis. J Clin Oncol. 2011;29:1479-1487.

3. Briganti A, Blute ML, Eastham JH, et al. Pelvic lymph node dissection in prostate cancer. Eur Urol. 2009;55:1251-1265.

4. Touijer KA, Ahallal Y, Guillonneau BD. Indications for and anatomical extent of pelvic lymph node dissection for prostate cancer: practice patterns of urooncologists in North America. Urol Oncol. 2013;31:1517-1521.

5. Yuh BE, Ruel NH, Mejia R, Wilson CM, Wilson TG. Robotic extended pelvic lymphadenectomy for intermediate- and high-risk prostate cancer. Eur Urol. 2012;61:1004-1010.

6. Winter A, Kneib T, Henke RP, Wawroschek F. Sentinel lymph node dissection in more than 1200 prostate cancer cases: rate and prediction of lymph node involvement depending on preoperative tumor characteristics. Int J Urol. 2014;21: 58-63.

7. Holl G, Dorn R, Wengenmair H, Weckermann D, Sciuk J. Validation of sentinel lymph node dissection in prostate cancer: experience in more than 2,000 patients. Eur J Nucl Med Mol Imaging. 2009;36:1377-1382.

8. FDA approves Lymphoseek to help locate lymph nodes in patients with certain cancers. J Nucl Med. 2013;54(5):20N.

9. Leong SP, Kim J, Ross M, et al. A phase 2 study of ${ }^{99 m}$ Tc-tilmanocept in the detection of sentinel lymph nodes in melanoma and breast cancer. Ann Surg Oncol. 2011;18:961-969.

10. Sondak VK, King DW, Zager JS, et al. Combined analysis of phase III trials evaluating $\left[{ }^{99 \mathrm{~m}} \mathrm{Tc}\right] \mathrm{tilmanocept}$ and vital blue dye for identification of sentinel lymph nodes in clinically node-negative cutaneous melanoma. Ann Surg Oncol. 2013;20:680-688.

11. Wallace AM, Han LK, Povoski SP, et al. Comparative evaluation of [ ${ }^{99 \mathrm{~m} T c]}$ tilmanocept for sentinel lymph node mapping in breast cancer patients: results of two phase 3 trials. Ann Surg Oncol. 2013;20:2590-2599.

12. Marcinow AM, Hall N, Byrum E, Teknos TN, Old MO, Agrawal A. Use of a novel receptor-targeted (CD206) radiotracer, ${ }^{99 \mathrm{~m}} \mathrm{Tc}$-tilmanocept, and SPECT/CT for sentinel lymph node detection in oral cavity squamous cell carcinoma: initial institutional report in an ongoing phase 3 study. JAMA Otolaryngol Head Neck Surg. 2013;139:895-902.

13. Stroup SP, Kane CJ, Farchshchi-Heydari S, et al. Preoperative sentinel lymph node mapping of the prostate using PET/CT fusion imaging and Ga-68-labeled tilmanocept in a dog model. Clin Exp Metastasis. 2012;29:673-680.

14. Wallace AM, Ellner SJ, Mendez J, et al. Minimally invasive sentinel lymph node mapping of the pig colon with Lymphoseek. Surgery. 2006;139:217-223.

15. Vera DR, Wisner ER, Stadalnik RC. Sentinel node imaging via a nonparticulate receptor-binding radiotracer. $J$ Nucl Med. 1997;38:530-535.

16. Vera DR, Wallace AM, Hoh CK, Mattrey RF. A synthetic macromolecule for sentinel node detection: [ ${ }^{99 m}$ Tc]DTPA-mannosyl-dextran. J Nucl Med. 2001;42: 951-959.

17. Hoh CK, Wallace AM, Vera DR. Preclinical studies of [ ${ }^{99 m}$ Tc]DPTA-mannosyldextran. Nucl Med Biol. 2003;30:457-464. 
18. Qin Z, Hall DJ, Liss MA, et al. Optimization via specific fluorescence brightness of a receptor-targeted probe for optical imaging and positron emission tomography of sentinel lymph nodes. J Biomed Opt. 2013;18:101315.

19. Warncke SH, Mattei A, Fuechsel FG, Z'Brun S, Krause T, Studer UE. Detection rate and operating time required for gamma probe-guided sentinel lymph node resection after injection of technetium-99m nanocolloid into the prostate with and without preoperative imaging. Eur Urol. 2007;52:126-132.

20. van der Poel HG, Buckle T, Brouwer OR, Valdes Olmos RA, van Leeuwen FW. Intraoperative laparoscopic fluorescence guidance to the sentinel lymph node in prostate cancer patients: clinical proof of concept of an integrated functional imaging approach using a multimodal tracer. Eur Urol. 2011;60:826-833.

21. Messing EM, Manola J, Sarosdy M, Wilding G, Crawford ED, Trump D. Immediate hormonal therapy compared with observation after radical prostatectomy and pelvic lymphadenectomy in men with node-positive prostate cancer. $\mathrm{N} \mathrm{Engl}$ J Med. 1999;341:1781-1788.

22. Joslyn SA, Konety BR. Impact of extent of lymphadenectomy on survival after radical prostatectomy for prostate cancer. Urology. 2006;68:121-125.

23. Passoni NM, Abdollah F, Suardi N, et al. Head-to-head comparison of lymph node density and number of positive lymph nodes in stratifying the outcome of patients with lymph node-positive prostate cancer submitted to radical prostatectomy and extended lymph node dissection. Urol Oncol. 2014;32: e21-e28.

24. Briganti A, Karnes JR, Da Pozzo LF, et al. Two positive nodes represent a significant cut-off value for cancer specific survival in patients with node positive prostate cancer: a new proposal based on a two-institution experience on 703 consecutive $\mathrm{N}+$ patients treated with radical prostatectomy, extended pelvic lymph node dissection and adjuvant therapy. Eur Urol. 2009;55:261-270.

25. Abdollah F, Suardi N, Cozzarini C, et al. Selecting the optimal candidate for adjuvant radiotherapy after radical prostatectomy for prostate cancer: a longterm survival analysis. Eur Urol. 2013;63:998-1008.
26. Weingärtner K, Ramaswamy A, Bittinger A, Gerharz EW, Voge D, Riedmiller H. Anatomical basis for pelvic lymphadenectomy in prostate cancer: results of an autopsy study and implications for the clinic. J Urol. 1996;156:1969-1971.

27. Yee DS, Katz DJ, Godoy G, et al. Extended pelvic lymph node dissection in robotic-assisted radical prostatectomy: surgical technique and initial experience. Urology. 2010;75:1199-1204.

28. Mattei A, Fuechsel FG, Bhatta Dhar N, et al. The template of the primary lymphatic landing sites of the prostate should be revisited: results of a multimodality mapping study. Eur Urol. 2008;53:118-125.

29. Wawroschek F, Vogt H, Weckermann D, Wagner T, Harzmann R. The sentinel lymph node concept in prostate cancer: first results of gamma probe-guided sentinel lymph node identification. Eur Urol. 1999;36:595-600.

30. Jeschke S, Beri A, Grull M, et al. Laparoscopic radioisotope-guided sentinel lymph node dissection in staging of prostate cancer. Eur Urol. 2008;53:126-132.

31. Briganti A, Chun FK, Salonia A, et al. Complications and other surgical outcomes associated with extended pelvic lymphadenectomy in men with localized prostate cancer. Eur Urol. 2006;50:1006-1013.

32. Weckermann D, Hamm M, Dorn R, Wagner T, Wawroschek F, Harzmann R. Sentinel lymph node dissection in prostate cancer. Experience after more than 800 interventions [in German]. Urologe A. 2006;45:723-727.

33. Jeschke S, Lusuardi L, Myatt A, Hruby S, Pirich C, Janetschek G. Visualisation of the lymph node pathway in real time by laparoscopic radioisotope- and fluorescenceguided sentinel lymph node dissection in prostate cancer staging. Urology. 2012; 80:1080-1086.

34. Brouwer OR, Buckle T, Vermeeren L, et al. Comparing the hybrid fluorescentradioactive tracer indocyanine green- ${ }^{99 \mathrm{~m}} \mathrm{Tc}$-nanocolloid with ${ }^{99 \mathrm{~m}} \mathrm{Tc}$-nanocolloid for sentinel node identification: a validation study using lymphoscintigraphy and SPECT/CT. J Nucl Med. 2012;53:1034-1040.

35. Liss MA, Stroup SP, Qin Z, Hall DJ, Kane CJ, Vera DR. Robotic-assisted fluorescentguided sentinel lymph node dissection in a preclinical model. Urology. In press. 